\title{
2-Photon Microscopy Imaging of Immune Cell Migration Across the Blood-Brain Barrier in the Spine During Induction of Acute and Chronic Injury
}

\author{
Y. Othman*, D.S. Barkauskas*, T. Evans**, J. Silver**, A.Y. Huang*
}

*Division of Pediatric Hematology/Oncology, Department of Pediatrics; **Department of Neuroscience, Case Western Reserve University School of Medicine, Cleveland, OH

Despite the improved outcome for pediatric leukemias, the most common tumor of childhood, gaps remain in our understanding of relapse and metastasis to the central nervous system (CNS). Thus, it is imperative to understand how tumors cross the BBB to help design new immune-mediated therapies against tumors in the CNS. By studying conditions such as autoimmune encephalomyelitis or acute spinal injury, we hope to gain a better insight of the blood brain barrier (BBB) physiology and mechanisms by which peripheral immune cells cross an otherwise impenetrable BBB.

We aim to demonstrate that a) focal breakdown of BBB integrity allows antigen presenting cell (APC) dendrite interaction with the vascular lumen; and, b) initial T cell recruitment to the CNS is the result of these APCs providing specific 'gates' across the BBB into the CNS.

We used 2-photon microscopy to map out the mechanisms mediating immune cell recruitment to and retention in the CNS of live experimental animals. We used Thy-1 YFP with ubiquitin CFP bone marrow chimeric mice to visualize neurons and immune cells, plus Cx3CR1 GFP mice to explicitly visualize microglia. The mice were either immunized with MOG peptide and pertussis toxin (PT) [1] or sustained an acute spinal injury [2]. After performing a laminectomy, we used intravital imaging as a way to monitor cellular recruitment to the spine over hours. The integrity of the BBB was monitored by infusing blood vessels with TRITC-dextran. Migration and distribution of blood-derived cells were quantified using tracking software, Imaris (BitPlane, Inc.).

Patchy BBB leakage and immune cell infiltration was visualized immediately after spinal cord injury (Figure 1). BBB leakage was evident by the increasing TRITCdextran labeled Cx3CR1+ microglia in the EAE model (Figure 2). This was followed by an increase in T cell and blood-derived MHC-II expressing APC infiltration and focal migration around sources of BBB leakage. As disease progressed, further breach in the BBB integrity was discerned as evidenced by axonal degeneration in the spinal cord injury model.

The recruitment and retention of the immune cells require the participation of both blood-derived and CNS-resident APCs. Targeting peri-vascular APCs may affect lymphocyte recruitment across the BBB. In future experiments, we will use a spinal window, still in development, to obtain long-term imaging data and monitor the migration of cells across the blood brain barrier into the spine parenchyma. 

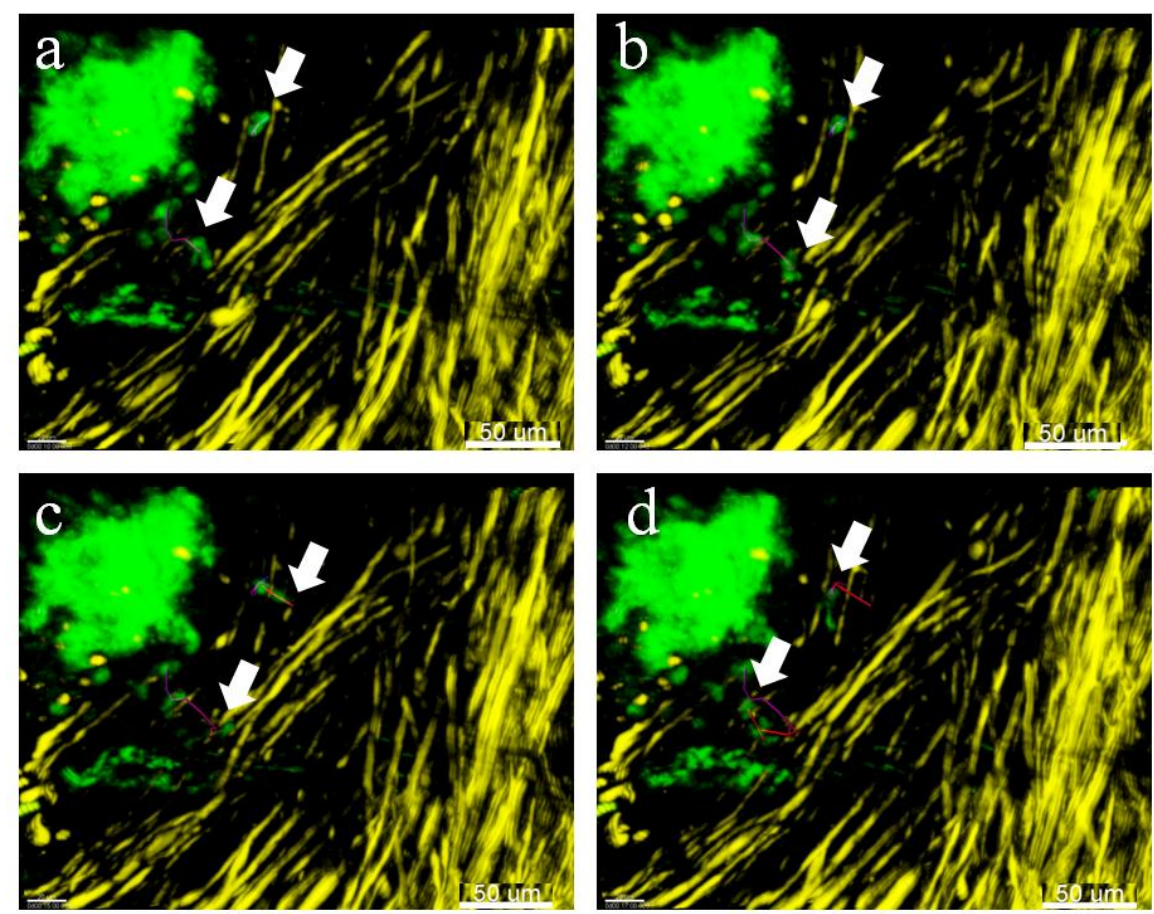

Figure 1: Spine imaging of an Ubiquitin CFP / Thy-1 half YFP chimera showing imaging of immune cells (green ) and neurons (yellow) in a live mouse. Movement of immune cells to an aseptic wound at time (a) $12 \mathrm{~min}$, (b) $14 \mathrm{~min}$, (c) $17 \mathrm{~min}$, (d) $19 \mathrm{~min}$, post injury.

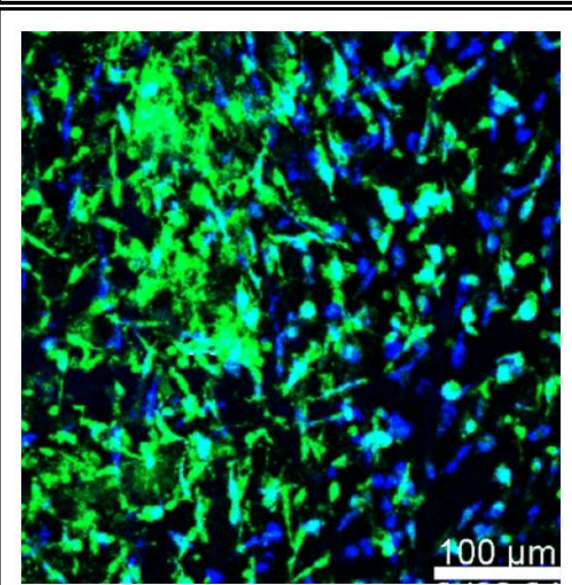

Figure 2: Imaging of Cx3CR1 GFP showing microglia(green) and dextran phagocytic cells (blue) in a live mouse.

\section{References:}

[1] I.M. Stromnes, J.M. Goverman, Nat Protocols, 1:4 (2006), 1810

[2] M.T. Fitch, et.al., J Neurosci. 1:19(1999), 8182

[3] This work was supported by grants from the St. Baldrick's Foundation, Rainbow Fellow Research Award Program, the Gabrielle's Angel Foundation, the Dana Foundation Brain- \& Immuno-Imaging Program, and the Cancer Research Foundation. 